ISSUES IN PUBLIC HEALTH

\title{
Adolescent girls and young women: Policy-to-implementation gaps for addressing sexual and reproductive health needs in South Africa
}

\author{
E L Davids, ${ }^{1,2}$ BPsych, MA, MPH, PhD; T Kredo, ${ }^{1,3} \mathrm{MB}$ ChB, MMed (Clin Pharm), Dip HIV Man (SA), PhD; A A M Gerritsen, ${ }^{4} \mathrm{PhD}$; \\ C Mathews, ${ }^{2,5} \mathrm{MSc}$, PhD; N Slingers, ${ }^{6} \mathrm{MB}$ ChB; M Nyirenda, ${ }^{7,8} \mathrm{BA}$, MPhil, PhD; F Abdullah, ${ }^{6,9} \mathrm{MB}$ ChB, FCPHM \\ ${ }^{1}$ Cochrane South Africa, South African Medical Research Council, Cape Town, South Africa \\ ${ }^{2}$ Adolescent Health Research Unit, Division of Child and Adolescent Psychiatry, Department of Psychiatry and Mental Health, \\ Faculty of Health Sciences, University of Cape Town, South Africa \\ ${ }^{3}$ Division of Clinical Pharmacology, Department of Medicine, Faculty of Medicine and Health Sciences, Stellenbosch University, Cape Town, South Africa \\ ${ }^{4}$ Epi Result, South Africa \\ ${ }^{5}$ Health Systems Research Unit, South African Medical Research Council, Cape Town, South Africa \\ ${ }^{6}$ Office of AIDS and TB Research, South African Medical Research Council, Cape Town, South Africa \\ ${ }^{7}$ Burden of Disease Unit, South African Medical Research Council, Cape Town, South Africa \\ ${ }^{8}$ School of Nursing and Public Health, University of KwaZulu-Natal, Durban, South Africa \\ ${ }^{9}$ Division of Infectious Diseases, Department of Internal Medicine, Faculty of Health Sciences, University of Pretoria, South Africa
}

Corresponding author: E L Davids (eugene.davids@mrc.ac.za)

\begin{abstract}
Sexual and reproductive health (SRH) services for adolescent girls and young women (AGYW) remain inadequate - both globally and in South Africa (SA). We systematically scoped the available policies and guidelines for SRH-related policy for AGYW in SA. We found many available policies and guidelines to address issues of family planning, HIV prevention and care and antenatal and maternal care. Despite the wealth of guidance, SA's high rates of pregnancy and HIV transmission continue unabated. Our policy review and analysis identified issues for researchers and policymakers to consider when developing and implementing programmes to improve SRH services. We suggest that considering national policies alongside evidence of what is effective, as well as contextual barriers to and enablers of strategies to address AGYW needs for SRH, are among the key steps to addressing the policy-to-implementation gap.
\end{abstract}

S Afr Med J 2020;110(9):855-857. https://doi.org/10.7196/SAMJ.2020.v110i9.14785

Sexual and reproductive health (SRH) services for adolescent girls and young women (AGYW) between the ages of 10 and 24 years remain inadequate in most parts of the world owing to individual, community, environmental and health system factors. ${ }^{[1]}$ In South Africa (SA), where there are colliding epidemics of HIV, sexually transmitted infections (STIs) and unintended pregnancies, these factors contribute to the lack of effective service provision and epidemic control. The SA Medical Research Council (SAMRC) is deploying an innovative financing and management model, a social impact bond, to promote SRH among AGYW. To support this effort, we reviewed the policy environment and identified critical issues for researchers and policymakers to consider when developing and implementing programmes in this field. ${ }^{[2]}$

\section{Sexual and reproductive health policies and guidelines}

Guidelines are documents that include recommendations for patient care, frameworks and standard operating procedures, ${ }^{[3]}$ whereas policies include 'decisions, plans, and actions that are undertaken to achieve specific health care goals within a society. ${ }^{[4]}$ In April 2019, we conducted a comprehensive search for national policies and guidelines regarding SRH outcomes relevant for the planned SAMRC programme for AGYW, aiming to address: (i) contraception uptake; (ii) pre-exposure prophylaxis (PrEP) initiation; (iii) antiretroviral treatment (ART); and (iv) enrolment and access to early antenatal care. We searched government websites, PubMed and Google and consulted experts in the field. We identified 4 relevant policies and 9 guidelines. Table 1 lists these policies and guidelines, grouped according to outcomes of the social impact bond for AGYW. ${ }^{[2]}$

SA needs policies and guidelines for effective interventions that empower people, particularly those who are most vulnerable, such as AGYW.$^{[5]}$ Mugabe ${ }^{[6]}$ suggests the following features of good policies and policymaking processes:

- informed by scientific knowledge or evidence

- open to public participation, transparent and allowing for local ownership by the people

- open to change and adaptation

- allowing for gradual, stepwise rather than radical implementation

- including implementation strategies aligned with current and existing capacity.

The SRH policies and guidelines (Table 1) are underpinned by some of the features Mugabe ${ }^{[6]}$ alludes to. The 2016 guidelines on PrEP have been informed by scientific knowledge and evidence from randomised controlled trials related to PrEP initiation and use, such as discussed by Choopanya et al..$^{[7]}$ and Grant et al. ${ }^{[8]}$ The maternity care guidelines are an example of guidelines open to change and adaptation throughout the process of implementation. 
Table 1. National policies/guidelines

Contraception to reduce pregnancy

National Adolescent Sexual and Reproductive Health and Rights Framework Strategy, 2014 - 2019

National Contraception and Fertility Planning Policy and Service Delivery Guidelines, 2012

National Youth Policy, 2015 - 2020

Integrated School Health Policy, 2012

National Adolescent and Youth Health Policy, 2017

Department of Basic Education Standard Operating Procedures for the Provision of Sexual and Reproductive Health Rights and Social Services in Secondary Schools, 2019

PrEP to reduce HIV seroconversion

National Strategic Plan for HIV, TB and STIs, 2017 - 2022

National Department of Health Guidelines for Expanding Combination Prevention and Treatment Options: Oral PrEP and Test and Treat, 2017

Southern African Guidelines on the Safe Use of PrEP in Persons at Risk of Acquiring HIV-1 Infection, 2016

Department of Basic Education Standard Operating Procedures for the Provision of Sexual and Reproductive Health Rights and Social Services in Secondary Schools, 2019

HIV testing and treatment to improve linkage to care and viral suppression

National Lesbian, Gay, Bisexual and Trans, Intersex HIV Framework, 2017 - 2022

National Consolidated Guidelines for the PMTCT of HIV and the Management of HIV in Children, Adolescents and Adults, 2015

Guideline: Adherence to Antiretroviral Therapy in Adolescents and Young Adults, 2017

Department of Basic Education National Policy on HIV, STIs and TB, 2017

Department of Basic Education Standard Operating Procedures for the Provision of Sexual and Reproductive Health Rights and Social Services in Secondary Schools, 2019

Antenatal care to improve pregnancy outcomes

National Department of Health Guidelines for Maternity Care in South Africa: A Manual for Clinics, Community Health Centres and

District Hospitals, 2016

PrEP = pre-exposure prophylaxis; $\mathrm{TB}=$ tuberculosis; $\mathrm{STI}=$ sexually transmitted infection; $\mathrm{PMTCT}$ = prevention of mother-to-child transmission.

They comprise a manual for clinics, community health centres and district hospitals, which is in its fourth edition and has seen changes and recommendations made over time. The draft policy on learner pregnancy is currently under discussion, and although it does not contain specific recommendations yet, it is indicative of a policy landscape open to refinement and gradual stepwise implementation. Many of the SRH policies and guidelines have reported the use of public participation during drafting, e.g. the National Adolescent and Youth Health Policy. However, one of the features of good policy and policymaking that remains a challenge, globally and in SA, is the gap between policy and implementation.

\section{Policy-implementation gap}

Despite the existence of evidence-informed policies and guidelines, AGYW continue to face high levels of pregnancy, HIV infection, school dropout and stigma, and limited access to SRH services. Among AGYW, the prevalence of pregnancy at the age of 15 years is $1.4 \%$, and increases to $25.0 \%$ by 19 years of age, with an average of 71 births per 1000 AGYW between 15 and 19 years of age. ${ }^{[9]}$ For AGYW, HIV is the second leading cause of death in the country, ${ }^{[9]}$ and these young women are 2 - 4 times more likely to be living with HIV than their male peers. ${ }^{[10]}$ AGYW are more likely to acquire HIV than their male peers, with numbers as high as $64 \%$ for AGYW. ${ }^{[5]}$ AGYW are benefiting from HIV services, but the gap needs to be closed between knowing your HIV status, accessing treatment and being virally suppressed. ${ }^{[5]}$ These figures point to the policy-implementation gap that exists in our country.

Many in SA are faced with poverty and unemployment, inequality and gender-based violence. These factors are known to impact on health and wellbeing, creating an exceedingly complex social environment in which to impact health, particularly for a vulnerable group such as AGYW. Given the complexity of the health and social system facing many AGYW, addressing the policy-implementation gap is both critical and challenging. Bridging the gap requires nimble and responsive implementation and evaluation of what is effective, using an implementation research lens. Implementation research is a field of research informing decisions about health policies, programmes and practices. It is related to all aspects of implementation, including upstream factors (context and barriers to effective implementation), policy landscape analysis (identifying gaps in policy implementation) and downstream factors (e.g. pragmatic trials to examine what is effective to address policy and practice needs). ${ }^{[11]}$

The National Adolescent and Youth Health Policy gives guidance on interventions to address prevention, testing and treatment for HIV. The policy suggests 'retain[ing] patients within healthcare services and support better adherence to medicines' and recommends youthfriendly services. Yet, many healthcare services are not youth friendly, particularly SRH services, with a knock-on effect of poor access to family planning and HIV prevention and care for AGYW. It is critical to understand how to address the policy-implementation gap and to work towards strengthening and supporting implementation. ${ }^{[12]}$ How exactly do we move from good policies and guidelines to successful implementation in a complex health system to yield the intended outcomes?

Recommendations to address the policy-implementation gap to improve SRH service provision include:

- Exploring and understanding the policy landscape. We have undertaken a policy review and the policies found can be further examined using Walt and Gilson's ${ }^{[13]}$ model of policy analysis. According to the model, there is a need to: (i) understand the context in which the policy is to be implemented, so that it is contextually relevant; (ii) unpack the content of the policy in a way that is clear and to the point, so that the intentions are understood 
by all; and (iii) delve into the implementation of the policy guided by the content and adaptation to the context in which it is to be executed, and to outline the process to be followed for successful implementation. However, it is essential to consider that the actors or agents of the health system are central throughout this process, as it is influenced by their everyday behaviours.

- Exploring and understanding the local context, including barriers to and enablers of AGYW accessing and receiving best care and services. This may take several approaches to 'diagnose' the issues. Qualitative research with the affected population, along with all the role players they interact with, may inform understanding about true needs, challenges and acceptable solutions.

- Considering the abovementioned factors, designing of the implementation strategy and evaluation approach. This should be done consultatively and be informed by available evidence of what is effective and the local context. Currently, there is little evidence on best implementation strategies for AGYW.

- Implementation of a robust, pragmatically designed evaluation. ${ }^{[1]}$ Ideally, this comprises using adaptive research designs that are flexible and responsive to the changing local context.

- Considering all the role players of the health system, as the success or failure of the policy implementation is shaped by their interaction. Thought should be given to capacity building and clear role delineation ${ }^{[12]}$ to address possible 'tensions' or conflicts between service providers and AGYW and the policy that might hinder roll-out of the implementation. In terms of adolescent SRH, this could address some of the issues found to be barriers to young women feeling uncomfortable with accessing SRH services due to limited capacity, or beliefs or motivations of healthcare workers regarding how best to execute the policies and guidelines.

- The need to foster cohesion among all role players, including researchers, funders, implementers and government departments, to work together to achieve the common goal of the policy or guideline.

Reflection and planning are needed for policy implementation, including consideration of benefits and harms, feasibility, acceptability and costs. ${ }^{[3,14]}$ Programmes such as the SA AGYW social impact bond have considered evidence of what works, the national policies in place and the contextual barriers to and enablers of strategies to address AGYW needs for SRH. Taken together, understanding the policy and social landscape can ensure that we can close the policyimplementation gap and make a difference to the lives of AGYW in SA.

\section{Declaration. None.}

Acknowledgements. None.

Author contributions. All authors contributed to the manuscript. ELD wrote the first draft and refined the manuscript, with inputs from all the authors.

Funding. The authors would like to acknowledge support from the SA Medical Research Council's Office of AIDS and TB Research.

Conflicts of interest. None.

1. Davids EL, Kredo T, Mathews C. Interventions for preventing unintended pregnancies among adolescents. S Afr Med J 2020;110(1):7-9. https://doi.org/10.7196/SAMJ.2019.v110i1.14281

2. Abdullah F, Naledi T, Nettleship E, et al. First social impact bond for the SAMRC: A novel financing strategy to address the health and social challenges facing adolescent girls and young women in South Africa. S Afr Med J 2019;109(11b):57-62. https://doi.org/10.7196/SAMJ.2019.v109i11b.14254

3. World Health Organization. Handbook for Guideline Development. 2nd ed. Geneva: WHO, 2014.

4. World Health Organization. Health topics: Health policy. 2020. https://www.who.int/topics/health policy/en/ (accessed 28 July 2020).

5. Joint United Nations Programme on HIV/AIDS (UNAIDS). We've Got the Power: Women, Adolescent Girls and the HIV Response. Geneva: UNAIDS, 2020.

6. Mugabe J. Characteristics of good public policy and policy-making. 2020. https://www.assaf.org.za/ files/2011/07/An-Overview-on-Good-Policy-Regimes-MUGABE.pdf (accessed 27 February 2020).

7. Choopanya K, Martin M, Suntharasamai P, et al. Antiretroviral prophylaxis for HIV infection in injecting drug users in Bangkok, Thailand (the Bangkok Tenofovir study): A randomised, double-
ing injecting drug users in Bangkok, Thailand (the Bangkok Tenofovir study): A randomised, double-
blind, placebo-controlled phase 3 trial. Lancet 2013;381(9883):2083-2090. https:///oi.org/10.1016/ blind, placebo-controlled
S0140-6736(13)61127-7

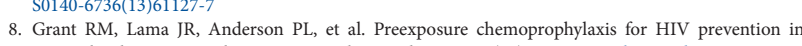
men who have sex with men. N Engl J Med 2010;363(27):2587-2599. https://doi.org/10.1056/ NEJMoa1011205

9. Statitics South Africa. Demographic Profile of Adolescents in South Africa. Pretoria: Stats SA, 2018.

10. Chimdindi N, Mthiyane N, Birdthistle I, et al. Persistently high incidence of HIV and poor servic uptake in adolescent girls and young women in rural KwaZulu-Natal, South Africa prior to DREAMS PLoS ONE 2018;13(10):e0203193. https://doi.org/10.1371/journal.pone.0203193

11. Peters DH, Tran NT, Adam T. Implementation Research in Health: A Practical Guide. Geneva: World Health Organization, 2013.

12. Hudson B, Hunter D, Peckham S. Policy failure and the policy-implementation gap: Can policy support programs help? Policy Design Pract 2019;2(1):1-14. https://doi.org/10.1080/25741292.2018.1540378

13. Walt G, Gilson L. Reforming the health sector in developing countries: The central role of policy Walt G, Gilson L. Reforming the health sector in developing countries: The central
analysis. Health Policy Plan 1994;9(4):353-370. https://doi.org/10.1093/heapol/9.4.353

14. Havers SM, Martin E, Wilson A, Hall L. Implementation of government-directed policy in the hospital setting: A modified Delphi study. Health Res Policy Syst 2019;17(91). https://doi.org/10.1186/s12961019-0500-8

Accepted 13 April 2020 